\title{
Astronomical bounds on the modified Chaplygin gas as a unified dark fluid model
}

\author{
Hang $\mathrm{Li}^{1}$, Weiqiang Yang ${ }^{2}$, and Liping Gai ${ }^{1}$ \\ 1 College of Medical Laboratory, Dalian Medical University, Dalian 116044, PR China \\ e-mail: lh@dmu.edu.cn \\ 2 Department of Physics, Liaoning Normal University, Dalian 116029, PR China
}

Received 12 July 2018 / Accepted 13 January 2019

\begin{abstract}
The modified Chaplygin gas could be considered to abide by the unified dark fluid model because the model might describe the past decelerating matter dominated era and at present time it provides an accelerating expansion of the Universe. In this paper, we have employed the Planck 2015 cosmic microwave background anisotropy, type-Ia supernovae, observed Hubble parameter data sets to measure the full parameter space of the modified Chaplygin gas as a unified dark matter and dark energy model. The model parameters $B_{s}, \alpha$, and $B$ determine the evolutional history of this unified dark fluid model by influencing the energy density $\rho_{\text {MCG }}=$ $\rho_{\mathrm{MCG} 0}\left[B_{s}+\left(1-B_{s}\right) a^{-3(1+B)(1+\alpha)}\right]^{1 /(1+\alpha)}$. We assumed the pure adiabatic perturbation of unified modified Chaplygin gas in the linear perturbation theory. In the light of Markov chain Monte Carlo method, we find that $B_{s}=0.727_{-0.039-0.079}^{+0.040+0.075}, \alpha=-0.0156_{-0.1380-0.2180}^{+0.0982+0.2346}$ $B=0.0009_{-0.0017-0.0030}^{+0.0018+0.0030}$ at $2 \sigma$ level. The model parameters $\alpha$ and $B$ are very close to zero and the nature of unified dark energy and dark matter model is very similar to cosmological standard model $\Lambda \mathrm{CDM}$.
\end{abstract}

Key words. dark energy - cosmological parameters

\section{Introduction}

In modern cosmology, many theoretical models have been used to explain the current accelerating expansion Amendola \& Tsujikawa (2010). Accelerating expansion of the Universe has been shown from the type Ia supernova (SNIa) observations Riess et al. (1998), Perlmutter et al. (1999) in 1998. Other more recent and updated observational results, including current cosmic microwave background (CMB) anisotropy measurement from Planck 2015 Planck Collaboration I (2016), Planck Collaboration XI (2016), Planck Collaboration XIII (2016), and the updated SNIa data sets from the Joint Light-curve Analysis (JLA) sample SDSS Collaboration (2014), also strongly support the present acceleration of the Universe. The latest release of Planck 2015 full-sky maps about the CMB anisotropies Planck Collaboration XIII (2016) indicates that baryon matter component occupies about $4 \%$ for total energy density, and about $96 \%$ energy density in the Universe is invisible which includes dark energy and dark matter. Dark energy (DE) as an exotic component with negative pressure drives the accelerated expansion of the Universe. And it is shown that DE takes up about two-thirds of the total energy density from cosmic observations. The remaining one third is dark matter (DM). In this way several theoretical DE models have already been constructed, for the reviews and papers, see Amendola \& Tsujikawa (2010), Weinberg (1989), Sahni \& Starobinsky (2000), Carroll (2001), Peebles \& Ratra (2003), Padmanabhan (2003), Copeland et al. (2006). However there exists another possibility that the invisible energy component is a unified dark fluid, in other words, a mixture of dark matter and dark energy.

If one treats the dark energy and dark matter as a unified dark fluid, the corresponding models have been put for- ward and studied in Kunz (2009), Capozziello et al. (2006), Hipolito-Ricaldi et al. (2010), Ananda \& Bruni (2006), Balbi et al. (2006), Xu et al. (2011, 2012a,b), Kamenshchik et al. (2001), Barrow (1988), Bento et al. (2002, 2004), Barreiro et al. (2008), Li et al. (2009), Wu \& Yu (2007), Park et al. (2010), Liang et al. (2011), Xu (2013a,b), Li \& Xu (2014a,b), Lu et al. (2008), Xu \& Lu (2010), Aviles \& Cervantes-Cota (2011), Luongo \& Quevedo (2012), Camera et al. (2009, 2011), Bertacca et al. (2008), Yang \& Xu (2013), Bruni et al. (2013), Piattella et al. (2010), Benaoum (2002), Ferreira \& Avelino (2015), Bilic et al. (2004), Beca \& Avelino (2007), Avelino et al. (2004, 2008, 2014), Beca et al. (2003), Avelino \& Ferreira (2015).

In these unified dark fluid models, the Chaplygin gas (CG) and its generalized models have been widely studied in order to explain the accelerating universe in Kamenshchik et al. (2001), Barrow (1988), Bento et al. (2002, 2004), Barreiro et al. (2008), $\mathrm{Li}$ et al. (2009), Wu \& Yu (2007), Park et al. (2010), Xu et al. (2011, 2012b), Liang et al. (2011), Xu (2013a,b), Li \& $\mathrm{Xu}$ (2014a,b), Lu et al. (2008), Xu \& Lu (2010), Benaoum (2002), Ferreira \& Avelino (2015), Bilic et al. (2004), Avelino et al. (2004, 2008, 2014), Beca \& Avelino (2007), Beca et al. (2003), Avelino \& Ferreira (2015). The most interesting property for this kind of scenarios is that two unknown dark sections-dark energy and dark matter can be unified by using an exotic equation of state. The original Chaplygin gas model can be obtained from the string Nambu-Goto action in the light cone coordinate Pedram \& Jalalzadeh (2008). In modern cosmology, the Chalygin gas could be considered as an dark fluid which might describe the past decelerating matter dominated era and at present time it provides an accelerating expansion of the Universe. For the generalized Chaplygin gas (GCG) model Bento et al. (2002), the equation of state 
Table 1. Mean values of model parameters with $1 \sigma$ and $2 \sigma$ errors and best-fit values from the joint constraint, whose results are obtained by the different combination of observational data sets $\mathrm{CMB}+\mathrm{CC}$ (second column), $\mathrm{CMB}+\mathrm{JLA}$ (third column), and CMB+JLA+CC (fourth column).

\begin{tabular}{|c|c|c|c|c|c|c|}
\hline Parameters & Means from $\mathrm{CMB}+\mathrm{CC}$ & Best fit & Means from CMB+JLA & Best fit & Means from $\mathrm{CMB}+\mathrm{JLA}+\mathrm{CC}$ & Best fit \\
\hline$\Omega_{\mathrm{b}} h^{2}$ & $0.02217_{-0.00016-0.00032}^{+0.00016+0.00032}$ & 0.02222 & $0.02219_{-0.00018-0.00032}^{+0.00016+0.00035}$ & 0.02226 & $0.02218_{-0.00016-0.00033}^{+0.00016+0.00034}$ & 0.02222 \\
\hline $100 \theta_{\mathrm{MC}}$ & $1.02863_{-0.00119-0.00283}^{+0.00164+0.00239}$ & 1.02889 & $1.02757_{-0.00123-0.000241}^{+0.001280 .00225}$ & 1.02775 & $1.02745_{-0.00095-0.00170}^{+0.00009+0.00180}$ & 1.02786 \\
\hline$\tau$ & $0.081_{-0.017-0.034}^{+0.017+0.033}$ & 0.075 & $0.080_{-0.017-0.033}^{+0.017+0.033}$ & 0.078 & $0.079_{-0.016-0.032}^{+0.016+0.032}$ & 0.082 \\
\hline$n_{\mathrm{s}}$ & $0.9688_{-0.0065-0.0109}^{+0.0054+0.0125}$ & 0.9644 & $0.9669_{-0.0074-0.00145}^{+0.0075+0.0135}$ & 0.9684 & $0.9672_{-0.0055-0.0106}^{+0.0056+0.0109}$ & 0.9686 \\
\hline $\ln \left(10^{10} A_{\mathrm{s}}\right)$ & $3.098_{-0.033-0.0067}^{+0.034+0.066}$ & 3.087 & $3.095_{-0.036-0.064}^{+0.032+0.065}$ & 3.094 & $3.093_{-0.032-0.064}^{+0.032+0.062}$ & 3.103 \\
\hline$B_{s}$ & $0.642_{-0.093-0.161}^{+0.097+0.171}$ & 0.639 & $0.730_{-0.047-0.0093}^{+0.047+0.089}$ & 0.731 & $0.727_{-0.039-0.079}^{+0.040+0.075}$ & 0.725 \\
\hline$\alpha$ & $-0.1688_{-0.2143-0.3080}^{+0.1456+0.3350}$ & -0.1576 & $0.0181_{-0.2199-0.3060}^{+0.01029+0.3953}$ & -0.0194 & $-0.0156_{-0.1380-0.02180}^{+0.0982+0.2346}$ & -0.1105 \\
\hline$B$ & $0.0016_{-0.0021-0.00036}^{+0.0017+0.0038}$ & 0.0007 & $0.0006_{-0.0023-0.00048}^{+0.00045}$ & 0.0007 & $0.0009_{-0.0017-0.0030}^{+0.10018+0.0030}$ & 0.0015 \\
\hline$H_{0}$ & $65.75_{-3.71-5.13}^{+2.60+6.18}$ & 64.74 & $68.07_{-3.10-5.22}^{+2.72+5.30}$ & 68.57 & $68.39_{-1.92-3.78}^{+2.10+3.68}$ & 68.07 \\
\hline Age/Gyr & $13.789_{-0.119-0.253}^{+0.133+0.229}$ & 13.850 & $13.778_{-0.235-0.375}^{+0.182+403}$ & 13.738 & $13.747_{-0.139-0.230}^{+0.119+0.250}$ & 13.721 \\
\hline
\end{tabular}

$p_{\mathrm{GCG}}=-A / \rho_{\mathrm{GCG}}^{\alpha}$ is characterized by two model parameters $A$ and $\alpha$. Ferreira \& Avelino (2015) considered an extended family of GCG models parameterized by three positive real parameters A, $\alpha$, and $\beta$, which, for two specific choices of $\beta[\beta=1$ and $\beta=(1+\alpha) /(2 \alpha)]$, is described by two different Lagrangians previously identified in the literature with GCG. Besides, a modified Chaplygin gas (MCG) model Benaoum (2002), which is a unified dark matter and dark energy model, is also an example of a dark fluid model. MCG model could be considered as the further generalization of GCG, whose equation of state is $p_{\mathrm{MCG}}=B \rho_{\mathrm{MCG}}-A / \rho_{\mathrm{MCG}}^{\alpha}$, with three degrees of freedom. This unified dark fluid model could also be adopted to explain the accelerating expansion of the Universe.

For the perturbation analysis of the $\mathrm{CG}$ and its generalized models, one might usually draw a different conclusion from the non-linear clustering effect or linear perturbation level. When one considers the non-linear clustering of the unified dark fluid, which may result in significant changes to the background evolution of the universe in unified dark energy models, see Bilic et al. (2004), Avelino et al. (2004, 2008, 2014), Beca \& Avelino (2007), Beca et al. (2003), and has recently been shown, the GCG may be consistent with current observational constraints over a wide region of parameter space, assuming that there is a sufficiently high level of non-linear clustering on small scales Avelino et al. (2014). In addition, when one focusses only on the cosmological effect in the linear perturbation theory, one could also use the recent observations to test the model parameter space of the CG and its generalized models, such as Xu et al. (2011, 2012b).

In order to constrain the model parameter space of MCG model in the linear perturbation theory, $\mathrm{Xu}$ et al. (2011) treated the dark energy and dark matter as a whole energy component, performed a global fitting on MCG model by the Markov chain Monte Carlo (MCMC) method by the observational data sets: CMB from WMAP-7 WMAP Collaboration (2011), BAO Percival et al. (2010), SNIa from Union2 SCP Collaboration (2010) data. The tight constraint had been obtained: $\alpha=0.000727_{-0.00140-0.00234}^{+0.00142+0.0039}, B=$ $0.000777_{-0.000302-0.000697}^{+0.000201+0.00015}$, and $B_{s}=0.782_{-0.0162-0.0329}^{+0.0163+0.0307}$ at $2 \sigma$ level. For the very small values of MCG parameters $\alpha$ and $B$, it was concluded that MCG is very close to $\Lambda$ cold dark matter $(\Lambda \mathrm{CDM})$ model. Moreover, Avelino \& Ferreira (2015) presents the much stronger constraints imposed in MCG model by the observed rotation curves of spiral galaxies, the results
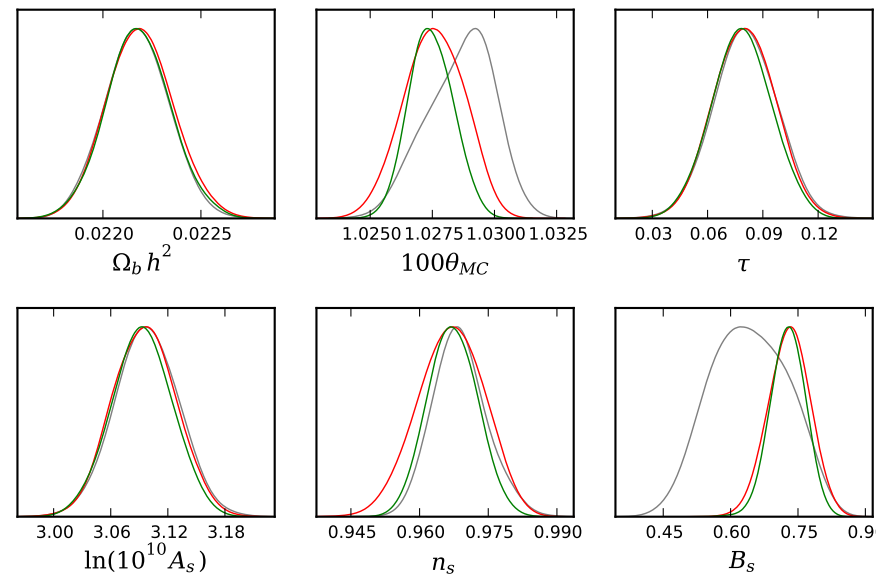

$n_{s}$
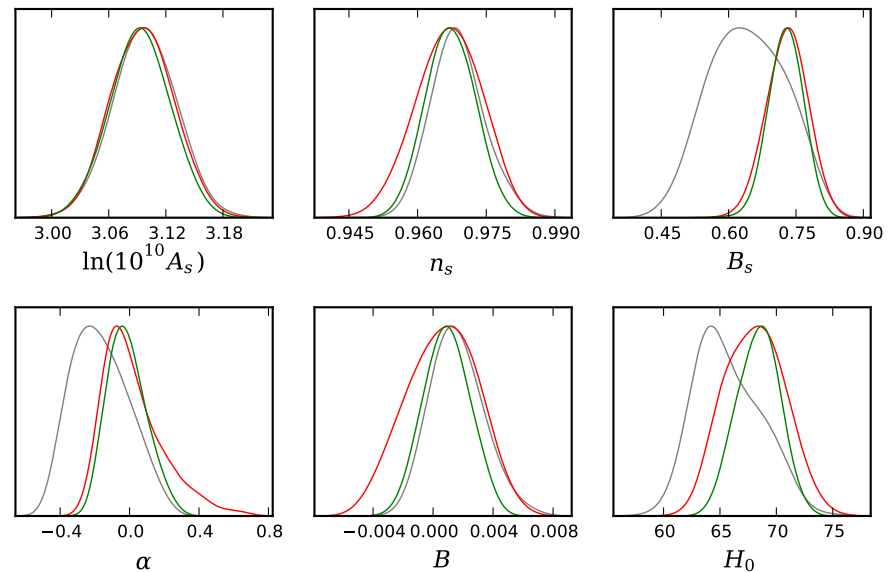

Fig. 1. One-dimensional marginalized distribution on individual parameters, whose results are obtained by the different combination of observational data sets $\mathrm{CMB}+\mathrm{CC}$ (gray line), $\mathrm{CMB}+\mathrm{JLA}$ (red line), and $\mathrm{CMB}+\mathrm{JLA}+\mathrm{CC}$ (green line).

show that $B<10^{-8}$ if the MCG is to be regarded as a unified dark fluid.

In the light of Xu et al. (2011), we test the parameter space of MCG model in the linear perturbation theory with the recently released data sets, CMB from Planck 2015 Planck Collaboration I (2016), Planck Collaboration XI (2016), Planck Collaboration XIII (2016), SNIa from JLA sample SDSS Collaboration (2014), and the observed Hubble parameter data Moresco et al. (2016), it is worth anticipating that a different observational constraint could be obtained.

This paper is organized as follows. In Sect. 2, we show the background and perturbation equations of MCG model when the pure adiabatic contribution has been considered. In Sect. 3, based on the MCMC method, the global fitting results of MCG model parameters are obtained by the joint observational data sets. Then, analyze the measurement results. Conclusion are drawn in the last section. 


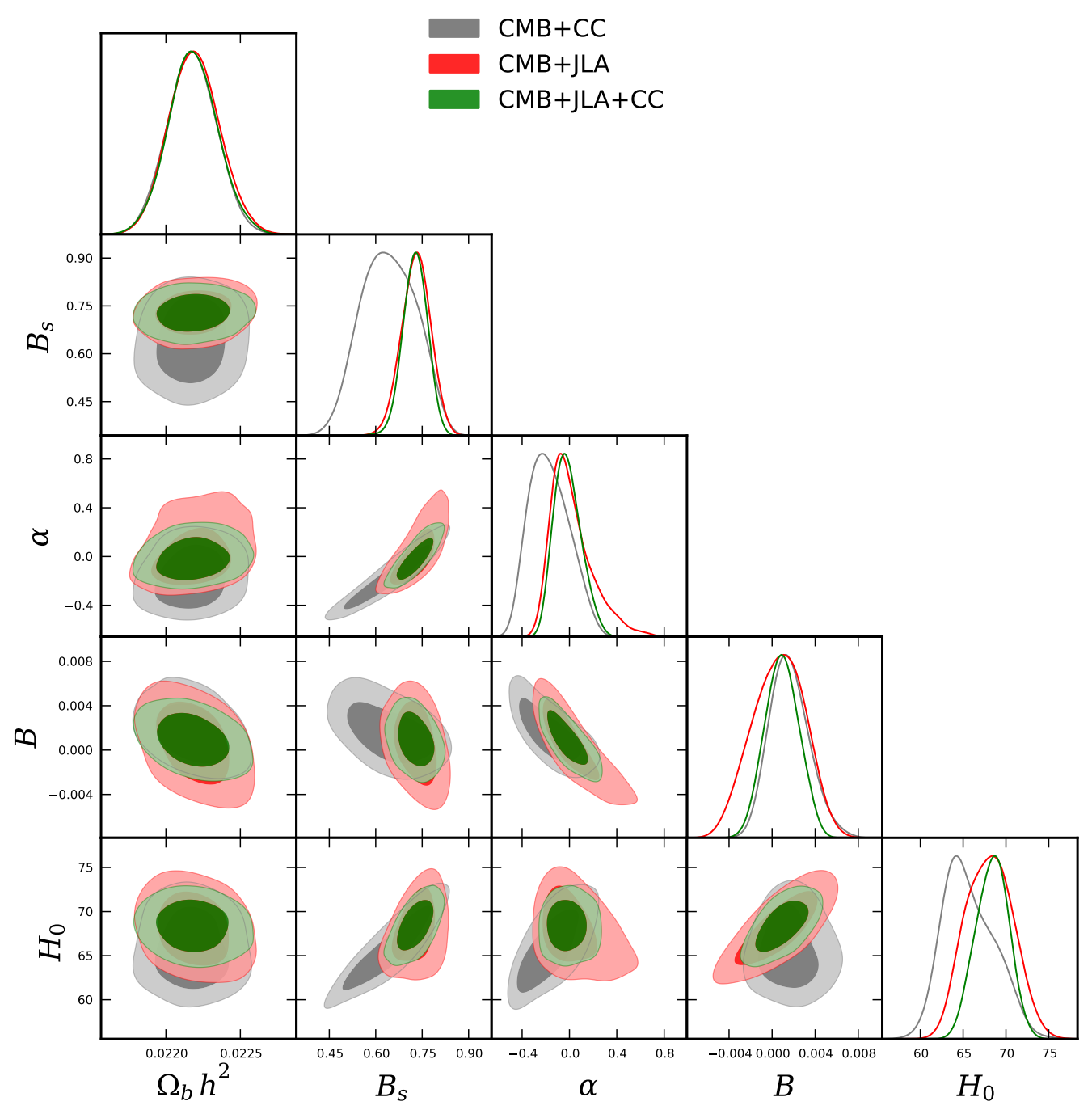

Fig. 2. One-dimensional marginalized distribution on individual parameters and two-dimensional contours of MCG model at $1 \sigma$ and $2 \sigma$ regions, whose results are obtained by the different combination of observational data sets $\mathrm{CMB}+\mathrm{JLA}$ (gray line or contour), $\mathrm{CMB}+\mathrm{CC}$ (red line or contour), and $\mathrm{CMB}+\mathrm{JLA}+\mathrm{CC}$ (green line or contour).

\section{The background and perturbation equations of modified Chaplygin gas model}

The MCG fluid is treated as a unified component in the Universe, its equation of state is

$p_{\mathrm{MCG}}=B \rho_{\mathrm{MCG}}-A / \rho_{\mathrm{MCG}}^{\alpha}$,

where $B, A$ and $\alpha$ are model parameters. It is obvious that the GCG model is recovered when the value of $B$ is zero.

By adopting the continuity equation, one could calculate the energy density of MCG fluid as

$\rho_{\mathrm{MCG}}=\rho_{\mathrm{MCG} 0}\left[B_{s}+\left(1-B_{s}\right) a^{-3(1+B)(1+\alpha)}\right]^{\frac{1}{1+\alpha}}$,

for $B \neq-1$, where $B_{s}=A /(1+B) \rho_{\mathrm{MCG} 0}^{1+\alpha}$. We take $B_{s}, B$ and $\alpha$ as MCG model parameters, and they would be constrained by the observational data sets. From Eq. (2), one can find that $0 \leq B_{s} \leq 1$ is demanded to keep the positivity of energy density. If $\alpha=0$ and $B=0$ in Eq. (2), the standard $\Lambda$ CDM model is recovered. Taking MCG as a unified dark fluid, the equation of state of MCG is

$w=B-(1+B) \frac{B_{s}}{B_{s}+\left(1-B_{s}\right) a^{-3(1+B)(1+\alpha)}}$,
In the flat Universe, one has the Friedmann equation

$\frac{H^{2}}{H_{0}^{2}}=\Omega_{\mathrm{b}} a^{-3}+\Omega_{r} a^{-4}+\Omega_{\mathrm{MCG}}\left[B_{s}+\left(1-B_{s}\right) a^{-3(1+B)(1+\alpha)}\right]^{\frac{1}{1+\alpha}}$

where $\Omega_{\mathrm{MCG}}=1-\Omega_{\mathrm{b}}-\Omega_{\mathrm{r}}, H$ and $H_{0}$ are the Hubble parameter and its present value, $\Omega_{\mathrm{b}}$ and $\Omega_{\mathrm{r}}$ are dimensionless energy density parameters of baryon and radiation.

Here, we consider the perturbation evolution of MCG fluid in order to explore the effects on the $\mathrm{CMB}$ anisotropic power spectrum. Under the assumption of pure adiabatic contribution to the perturbations Xu et al. (2012b), the sound speed of MCG is

$c_{\mathrm{s}}^{2}=\frac{\delta p}{\delta \rho}=\frac{\dot{p}}{\dot{\rho}}=-\alpha w+(1+\alpha) B$.

According to the conservation of energy-momentum tensor $T_{v ; \mu}^{\mu}=0$, ignoring the shear perturbation, one could the deduce the perturbation equations of density contrast and velocity divergence for MCG in the linear perturbation theory Xu et al. (2012b)

$\dot{\delta}_{\mathrm{MCG}}=-(1+w)\left(\theta_{\mathrm{MCG}}+\frac{\dot{h}}{2}\right)-3 \mathcal{H}\left(c_{\mathrm{s}}^{2}-w\right) \delta_{\mathrm{MCG}}$ 
$\dot{\theta}_{\mathrm{MCG}}=-\mathcal{H}\left(1-3 c_{\mathrm{s}}^{2}\right) \theta_{\mathrm{MCG}}+\frac{c_{\mathrm{s}}^{2}}{1+w} k^{2} \delta_{\mathrm{MCG}}-k^{2} \sigma_{\mathrm{MCG}}$

where the dot denotes the derivative of conformal time, the notations follow Ma and Bertschinger Ma \& Berschinger (1995). In our calculation, the shear perturbation $\sigma_{\mathrm{MCG}}=0$ is assumed and the adiabatic initial conditions are used.

\section{Observational data sets and methodology}

In this section we first describe the astronomical data with the statistical technique to constrain the MCG scenarios and the results of the analyses. We include the following sets of astronomical data.

- CMB: We used CMB data from the Planck 2015 measurements Planck Collaboration I (2016) and Planck Collaboration XI (2016), where we combined the full likelihoods $C_{l}^{\mathrm{TT}}, C_{l}^{\mathrm{EE}}, C_{l}^{\mathrm{TE}}$ in addition with low- $l$ polarization $C_{l}^{\mathrm{TE}}+C_{l}^{\mathrm{EE}}+C_{l}^{\mathrm{BB}}$, which notationally is same with "PlanckTT, TE, EE + lowP" of Planck Collaboration XI (2016).

- JLA: This is the supernovae Type Ia sample that contains 740 data points spread in the redshift interval $z \in[0.01,1.30]$ SDSS Collaboration (2014). This low-redshift sample is the first indication for an accelerating universe.

- Cosmic chronometers (CC): The Hubble parameter measurements from most old and passively evolving galaxies, known as cosmic chronometers have been considered to be potential candidates to probe the nature of dark energy due to their model-independent measurements. For a detailed description on howthe Hubble parameter values can be measured at different redshifts through this $\mathrm{CC}$ approach, and its usefulness, we refer to Moresco et al. (2016). Here, we have used 30 measurements of the Hubble parameter at different redshifts within the range $0<z<2$.

So the total likelihood $\chi^{2}$ can be constructed as

$\chi^{2}=\chi_{\mathrm{CMB}}^{2}+\chi_{\mathrm{JLA}}^{2}+\chi_{\mathrm{CC}}^{2}$.

In order to extract the observational constraints of the MCG scenarios, we use the publicly available MCMC package COSMOMC Lewis \& Bridle (2002) equipped with a convergence diagnostic followed by the Gelman and Rubin statistics, which includes the CAMB code to calculate the CMB power spectrum. We modified this code for the MCG model with the perturbation of unified dark fluid. We have used the following eightdimensional parameter space

$P \equiv\left\{\omega_{\mathrm{b}}, 100 \theta_{\mathrm{MC}}, \tau, B_{s}, \alpha, B, n_{\mathrm{s}}, \log \left[10^{10} A_{\mathrm{s}}\right]\right\}$

where $\Omega_{\mathrm{b}} h^{2}$ stands for the density of the baryons and dark matter, $100 \theta_{\mathrm{MC}}$ refers to the ratio of sound horizon and angular diameter distance, $\tau$ indicates the optical depth, $B_{s}, \alpha$, and $B$ are three added parameters of MCG model, $n_{\mathrm{s}}$ is the scalar spectral index, and $A_{\mathrm{s}}$ represents the amplitude of the initial power spectrum. The pivot scale of the initial scalar power spectrum $k_{s 0}=0.05 \mathrm{Mpc}^{-1}$ is used. In the MCMC analysis, we took the following priors to model parameters: $\Omega_{\mathrm{b}} h^{2} \in[0.005,0.1]$, $\Theta_{S} \in[0.5,10], \tau \in[0.01,0.8], B_{s} \in[0,1], \alpha \in[-1,1]$, $B \in[-1,1], n_{\mathrm{s}} \in[0.5,1.5]$ and $\log \left[10{ }^{10} A_{\mathrm{s}}\right] \in[2.7,4]$.

\section{Analysis the fit results}

In this section we summarize the main observational results extracted from the MCG unified model by using the three
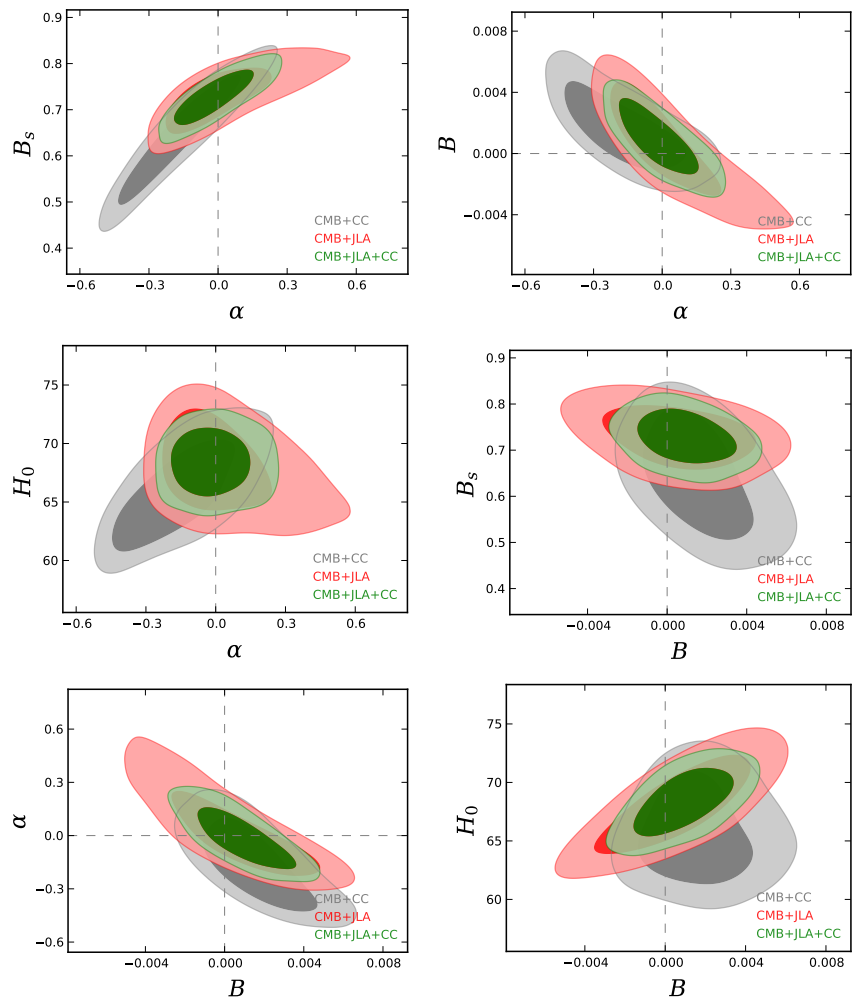

Fig. 3. Two-dimensional contours of MCG model at $1 \sigma$ and $2 \sigma$ regions for the parameters pair, the dashed vertical line is corresponding to the parameter $\alpha=0$ or $B=0$, whose results are obtained by the different combination of observational data sets $\mathrm{CMB}+\mathrm{CC}$ (gray contour), $\mathrm{CMB}+\mathrm{JLA}$ (red contour), and $\mathrm{CMB}+\mathrm{JLA}+\mathrm{CC}$ (green contour).

different combined observational data, $\mathrm{CMB}+\mathrm{JLA}, \mathrm{CMB}+\mathrm{CC}$, $\mathrm{CMB}+\mathrm{JLA}+\mathrm{CC}$, described in Sect. 3 above. In Table 1 we summarize the main results of global fitting results, at the first sight, the combination $\mathrm{CMB}+\mathrm{CC}$ provide a slightly different constraint results of the model parameters, comparing with CMB+JLA and $\mathrm{CMB}+\mathrm{JLA}+\mathrm{CC}$. One could see that for the combination $\mathrm{CMB}+\mathrm{CC}$, both the mean value and best-fit value of the parameter $B_{s}$ and $H 0$ are lower, meanwhile the other two combination $\mathrm{CMB}+\mathrm{JLA}$ and $\mathrm{CMB}+\mathrm{JLA}+\mathrm{CC}$ show the higher the mean value of best-fit value $B_{s}$ and $H_{0}$. Testing the result of the parameter $\alpha$, the observational data sets favor a negative value, except for the mean value from $\mathrm{CMB}+\mathrm{JLA}$. Testing the result of the parameter $B$, the observational data sets support a very small and positive value, its order is $10^{-3}$ or $10^{-4}$. All the results of three different observational combination support that the parameters $\alpha$ and $B$ are very close to zero, it means that the nature of MCG model is very similar to cosmological standard model $\Lambda \mathrm{CDM}$. In order to clearly and visually show the difference between different combination, we have also plotted the one-dimensional marginalized posterior distribution on individual parameters in Fig. 1 which are fully corresponding to the Table 1 . It is easy to see that for the parameters $\Omega_{b} h^{2}, \tau, \log \left[10^{10} A_{\mathrm{s}}\right], n_{\mathrm{s}}$ are almost the same for the three different combination of data sets, however one would see the obvious difference in the mean values with error bars for the parameters $100 \theta_{\mathrm{MC}}, B_{s}, \alpha, B, H_{0}$ ( $H_{0}$ is a derived parameter). Besides, the combination $\mathrm{CMB}+\mathrm{JLA}$ and $\mathrm{CMB}+\mathrm{JLA}+\mathrm{CC}$ provide the nearly identical results, and these two cases' global fittings show us their somewhat tighter constraint than that of $\mathrm{CMB}+\mathrm{CC}$. For the MCG model, the $\mathrm{CC}$ data set could not improve the constraint results. 

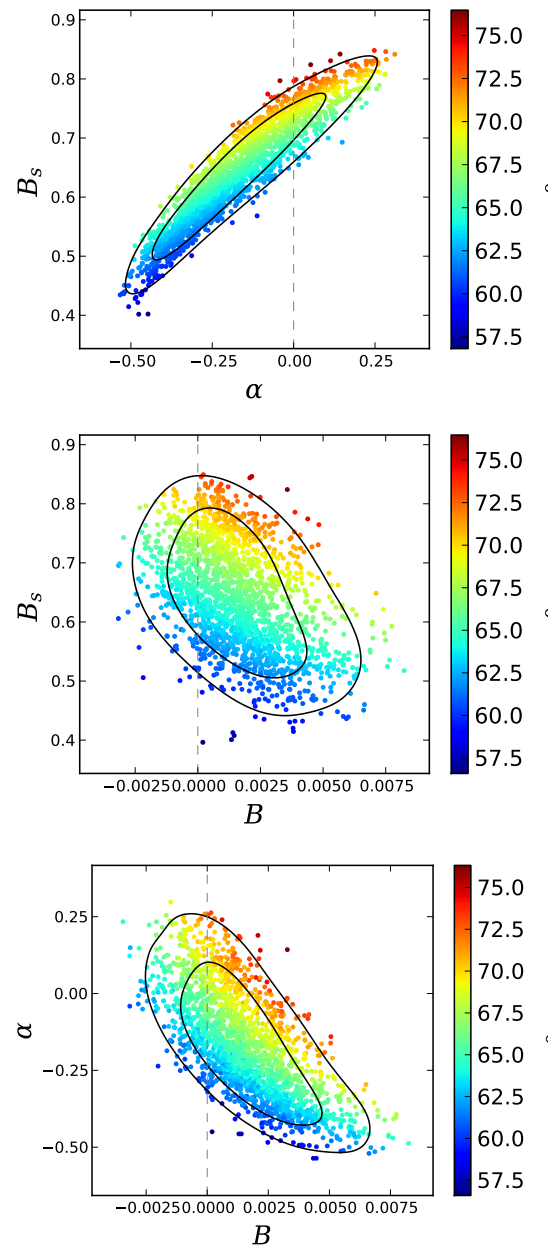
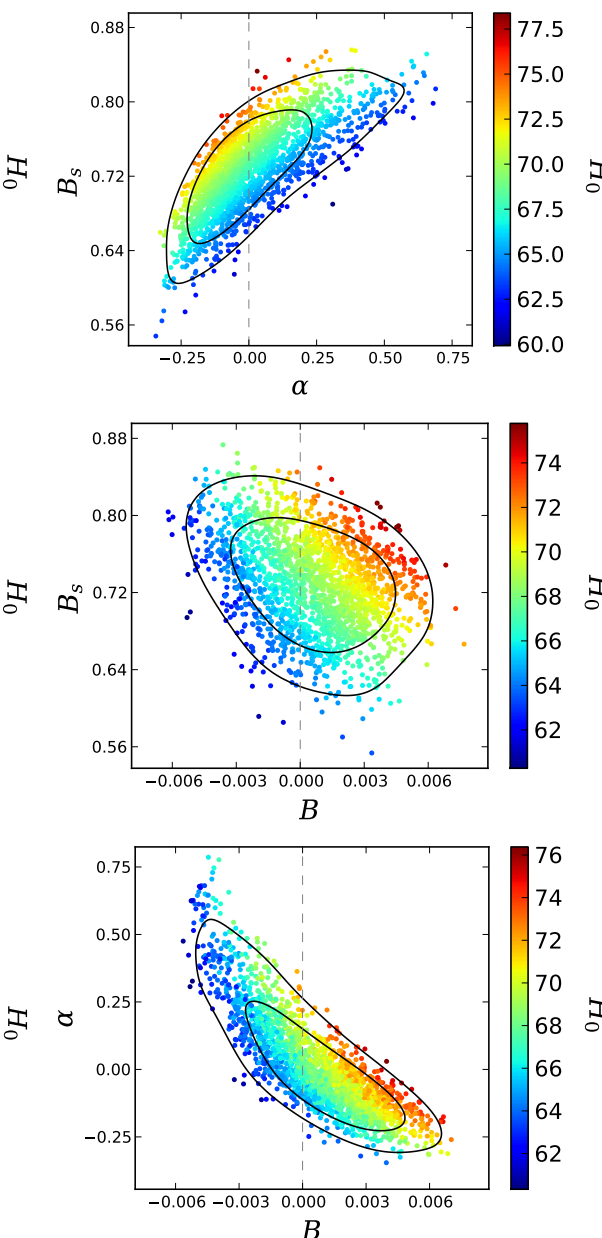
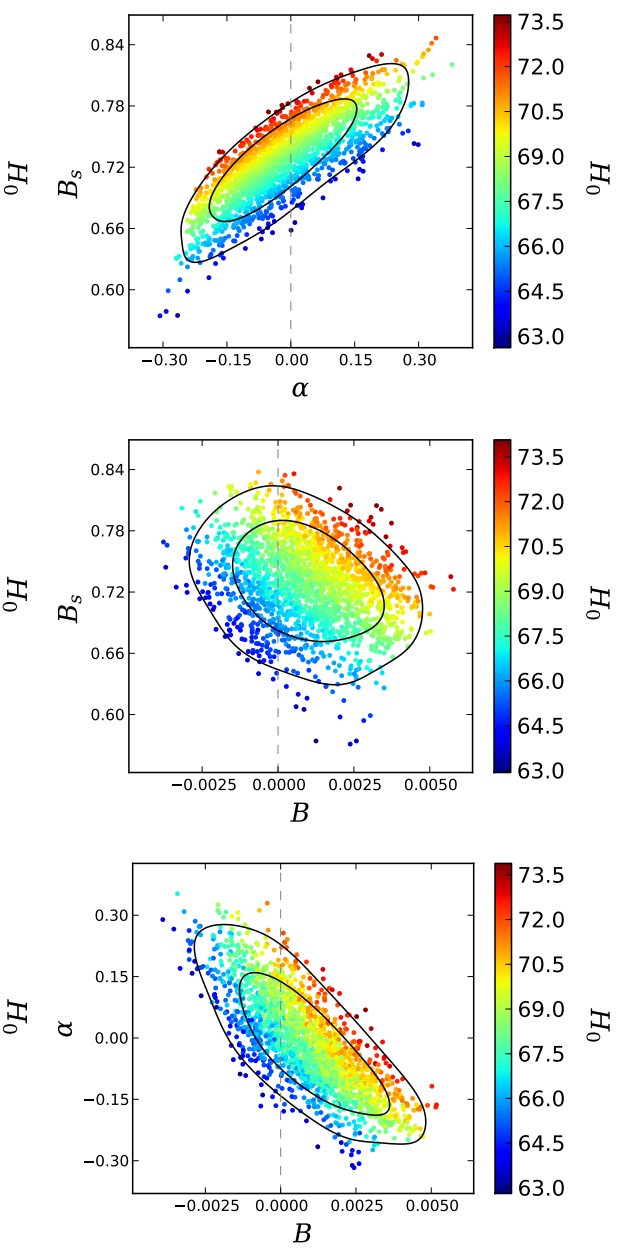

Fig. 4. Three-dimensional figures with colors of MCG model between the parameters pair $B_{s}, \alpha, B$ and derived parameter $H_{0}$ for three different observational combination, the dashed vertical line is corresponding to the parameter $\alpha=0$ or $B=0$, whose results, from left to right, are obtained by the different combination of observational data sets $\mathrm{CMB}+\mathrm{CC}$ (left panel), $\mathrm{CMB}+\mathrm{JLA}$ (middle panel), and CMB+JLA+CC (right panel).
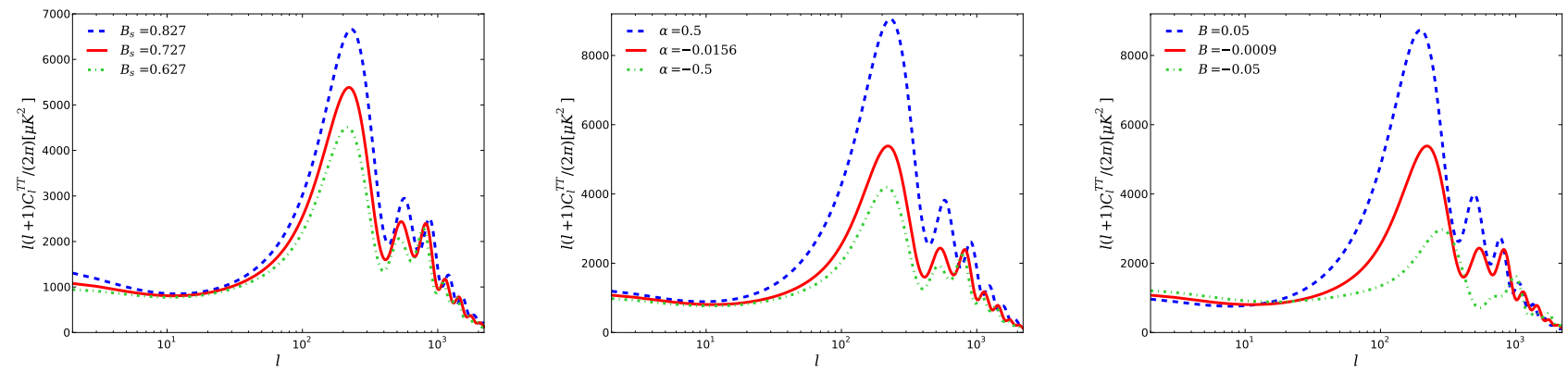

Fig. 5. Effects on CMB temperature power spectra for the different values of the model parameter $\alpha$ and $B_{s}$. In the left figure, the blue dashed, red solid, and green dotted-dashed lines are for $B_{s}=0.827,0.727,0.627$, respectively; in the middle figure, the blue dashed, red solid, and green dotted-dashed lines are for $\alpha=0.5,-0.0156,-0.5$, respectively; in the right figure, the blue dashed, red solid, and green dotted-dashed lines are for $B=0.05,-0.0009,-0.05$, respectively; the other relevant parameters are fixed with the mean values of CMB $+\mathrm{JLA}+\mathrm{CC}$ as shown in Table 1 .

Then, we also show the one-dimensional marginalized posterior distribution and two-dimensional marginalized posterior distribution contours which include the MCG model parameters $B_{s}$, $\alpha, B$ are shown in Fig. 2, one could see that the parameter $\Omega_{\mathrm{b}} h^{2}$ owns the vague correlation with the other parameters, the MCG model parameters $B_{s}$ and $\alpha$ show the obvious positive correlation, meanwhile, the parameters $B_{s}$ and $B, \alpha$ and $B$ show the negative correlation, both $B_{s}$ and $B$ show the distinct positive correlation with the derived parameter $H_{0}$, but the correlation between $\alpha$ and $H_{0}$ is not clear. Here we also extract the two-dimensional contours for several parameter pairs $B_{s}-\alpha$, $B-\alpha, H_{0}-\alpha, B_{s}-B, \alpha-B$, and $H_{0}-B$ in Fig. 3, and the three-dimensional figures with colors between the parameter pairs $B_{s}-\alpha, B_{s}-B$, and $\alpha-B$ and the present Hubble parameter $H_{0}$ in Fig. 4. From the one-dimensional, two-dimensional, threedimensional figures and the Table 1, one could see that the combinations $\mathrm{CMB}+\mathrm{JLA}$ and $\mathrm{CMB}+\mathrm{JLA}+\mathrm{CC}$ show better constraint results than $\mathrm{CMB}+\mathrm{CC}$. The recent observational data sets, especially the combination $\mathrm{CMB}+\mathrm{JLA}$ and $\mathrm{CMB}+\mathrm{JLA}+\mathrm{CC}$, support both the negative and positive values of the parameters $\alpha$ and $B$ 
which is close to zero, and testing the results of the combination $\mathrm{CMB}+\mathrm{JLA}+\mathrm{CC}$, the mean value and best-fit value of $\alpha$ is negative, ones of $B$ positive. The mean value of parameter $B_{s}$ would be larger than 0.7 from both the combination CMB+JLA and $\mathrm{CMB}+\mathrm{JLA}+\mathrm{CC}$, the mean value of parameter $H_{0}$ would be larger than $68 \mathrm{~km} \mathrm{~s}^{-1} \mathrm{Mpc}^{-1}$ from both the combination CMB+JLA and $\mathrm{CMB}+\mathrm{JLA}+\mathrm{CC}$.

In order to well understand the effects of model parameters on the CMB temperature anisotropic power spectra, we plot the temperature power spectra of $\mathrm{CMB}$, where one of three model parameters $B_{s}, \alpha, B$ varies, where the other relevant parameters are fixed to their mean values as listed in Table 1 . The panels of Fig. 5 show, respectively, the effects of parameters $B_{s}, \alpha$, and $B$ on CMB power spectra. The model parameter $\alpha$ modifies the power law of the energy density of MCG, then it makes the gravity potential evolution at late epoch of the universe. As results, one can see the integrated Sachs-Wolfe (ISW) effect on the large scale as shown in the left panel of Fig. 5. In the early epoch, MCG behaves like cold dark matter with almost zero equation of state and speed of sound $c_{\mathrm{s}}^{2}$, therefore the variation of the values of $\alpha$ will change the ratio of energy densities of the effective cold dark matter and baryons. One can read the corresponding effects from the variation of the first and the second peaks of CMB power spectra. For various values of the model parameter $B_{s}$, decreasing the values of $B_{s}$, which is equivalent to increase the value of effective dimensionless energy density of cold dark matter, causes matter and radiation to become equal earlier, therefore decreasing the sound horizon. As a result, the first peak is depressed. Changing the value of the model parameter $B$, changes both the peak height and peak location. Decreasing the values of $B$ decreases the peak height, and the peak location moves to the high- $l$ region, because changing $B$ causes the proportional relation of dark matter and baryons to change. Figure 6 shows CMB power spectra and its corresponding raito with mean values listed in Table 1 for MCG model and $\Lambda \mathrm{CDM}$ model. The black dashed line represents the $\Lambda$ CDM model with mean values taken from Planck 2015 Planck Collaboration XIII (2016) constraint results. One can see that the MCG model matches both observational data points and the $\Lambda$ CDM model well.

\section{Summary}

In this paper, we have employed the Planck 2015 cosmic microwave background anisotropy, type-Ia supernovae, and observed Hubble parameter data sets to measure the full parameter space of the modified Chaplygin gas as a unified dark matter and dark energy model. The parameters $B_{s}, \alpha$, and $B$ determine the evolutionary history of this unified dark fluid model by influencing the energy density $\rho_{\mathrm{MCG}}=\rho_{\mathrm{MCG} 0}\left[B_{s}+(1-\right.$ $\left.\left.B_{S}\right) a^{-3(1+B)(1+\alpha)}\right]^{1 /(1+\alpha)}$. We assumed the pure adiabatic perturbation of unified modified Chaplygin gas. In the light of the MCMC method, we find that at $2 \sigma$ level, $B_{s}=0.642_{-0.093-0.161}^{+0.097+0.171}$, $\alpha=-0.1688_{-0.2143-0.3080}^{+0.1456+0.3350}, B=0.0016_{-0.0021-0.0036}^{+0.0017+0.0038}$ from CMB+ $\mathrm{CC}, B_{s}=0.730_{-0.047-0.093}^{+0.047+0.089}, \alpha=0.0181_{-0.2199-0.3060}^{+0.1029+0.3953}, B=$ $0.0006_{-0.0023-0.0048}^{+0.0028+0.0045}$ from CMB+JLA, $B_{s}=0.642_{-0.093-0.161}^{+0.097+0.171}$, $\alpha=-0.1688_{-0.2143-0.3080}^{+0.1456+0.3350}$, and $B=0.0016_{-0.0021-0.0036}^{+0.0017+0.0038}$ from $\mathrm{CMB}+\mathrm{JLA}+\mathrm{CC}$. A tight constraint is obtained as shown in Table 1 and Fig. 2. The results of all three different observational combinations support the idea that the parameters $\alpha$ and $B$ are very close to zero, the order of $\alpha$ is $10^{-2}$, the order of $B$ is $10^{-4}$. This means that the nature of the MCG model is very similar to the cosmological standard model $\Lambda \mathrm{CDM}$. The MCG model can
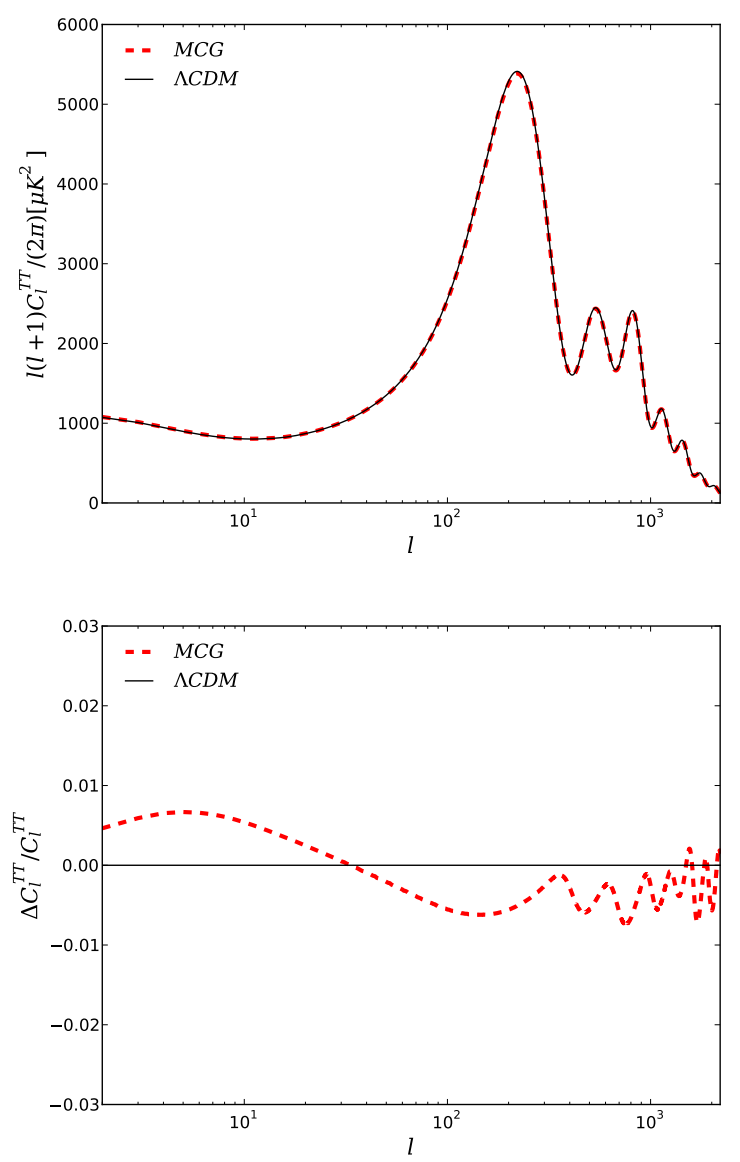

Fig. 6. CMB temperature power spectrum vs. multiple moment $l$, the red solid line is for the unified dark fluid model with mean values of $\mathrm{CMB}+\mathrm{JLA}+\mathrm{CC}$ as shown in Table 1 , the black dashed line is for the $\Lambda C D M$ model with the same observational combination.

well match both observational data points and the $\Lambda$ CDM model. Currently available data sets of CMB, JLA, and CC could not distinguish MCG model from $\Lambda$ CDM model.

Here we have not used the baryon acoustic oscillation (BAO) data to test the MCG model parameter space, because if one wants to use the BAO data to test a unified dark fluid model, one needs to know the sound horizon at the redshift of drag epoch $z_{d}$. Usually, $z_{d}$ is obtained by using the accurate fitting formula Eisenstein \& $\mathrm{Hu}$ (1998) which is valid if the matter scalings $\rho_{\mathrm{b}} \propto a^{-3}$ and $\rho_{\mathrm{c}} \propto a^{-3}$ are respected. Obviously, this is not true for the unified dark fluid model. One might reconsider the fiducial cosmology according to the analysis of Percival et al. (2010), Hamann et al. (2010), and Xu et al. (2012b), this will be an important research field in our future work.

Acknowledgements. H. Li's work is supported by the National Natural Science Foundation of China under Grant No. 81601855. W. Yang's work is supported by the National Natural Science Foundation of China under Grants No. 11705079 and 11647153 .

\section{References}

Amendola, L., \& Tsujikawa, S. 2010, Dark Energy: Theory and Observations (New York: Cambridge University Press)

Ananda, K. N., \& Bruni, M. 2006, Phys. Rev. D, 74, 023523

Aviles, A., \& Cervantes-Cota, J. L. 2011, Phys. Rev. D, 84, 083515

Avelino, P. P., \& Ferreira, V. M. C. 2015, Phys. Rev. D, 91, 083508

Avelino, P. P., Beca, L. M. G., de Carvalho, J. P. M., Martins, C. J. A. P., \& Copeland, E. J. 2004, Phys. Rev. D, 69, 041301 
Hang Li et al.: Astronomical bounds on the modified Chaplygin gas as a unified dark fluid model

Avelino, P. P., Beca, L. M. G., \& Martins, C. J. A. P. 2008, Phys. Rev. D, 77, 063515

Avelino, P. P., Bolejko, K., \& Lewis, G. F. 2014, Phys. Rev. D, 89, 103004 Balbi, A., Bruni, M., \& Quercellini, C. 2006, Phys. Rev. D, 76, 103519

Barrow, J. D. 1988, Nucl. Phys. B, 310, 743

Barreiro, T., Bertolami, O., \& Torres, P. 2008, Phys. Rev. D, 78, 043530

Beca, L. M. G., \& Avelino, P. P. 2007, MNRAS, 376, 1169

Bento, M. C., Bertolami, O., \& Sen, A. A. 2002, Phys. Rev. D, 66, 043507

Bento, M. C., Bertolami, O., \& Sen, A. A. 2004, Phys. Rev. D, 70, 083519

Benaoum, H. B., ArXiv e-prints [arXiv:hep-th/0205140]

Bertacca, D., Bartolo, N., Diaferio, A., \& Matarrese, S. 2008, JCAP, 10, 023

Beca, L. M. G., Avelino, P. P., De Carvalho, J. P. M., \& Martins, C. J. A. P. 2003 Phys. Rev. D, 67, 101301

Bilic, N., Lindebaum, R. J., Tupper, G. B., \& Violier, R. D. 2004, JCAP, 11, 008

Bruni, M., Lazkoz, R., \& Rozas-Fernandez, A. 2013, MNRAS, 431, 2907

Carroll, S. M. 2001, Liv. Rev. Relat., 4, 1

Camera, S., Bertacca, D., Diaferio, A., et al. 2009, MNRAS, 399, 1995

Camera, S., Kitching, T. D., Heavens, A. F., et al. 2011, MNRAS, 415, 399

Capozziello, S., Nojiri, S., \& Odintsov, S. D. 2006, Phys. Lett. B, 632, 597

Copeland, E. J., Sami, M., \& Tsujikawa, S. 2006, Int. J. Mod. Phys. D, 15, 1753

Eisenstein, D. J., \& Hu, W. 1998, ApJ, 496, 605

Ferreira, V. M. C., \& Avelino, P. P. 2015, Phys. Rev. D, 98, 043515

Hamann, J., Hannestad, S., Raffelt, G. G., \& Wong, Y. Y. Y. 2010, JCAP, 07, 022

Hipolito-Ricaldi, W. S., Velten, H. E. S., \& Zimdahl, Z. 2010, Phys. Rev. D, 82, 063507

Kamenshchik, A. Y., Moschella, U., \& Pasquier, V. 2001, Phys. Lett. B, 511, 265 Kunz, M. 2009, Phys. Rev. D, 80, 123001

Lewis, A., \& Bridle, S. 2002, Phys. Rev. D, 66, 103511

Li, W., \& Xu, L. 2014a, Eur. Phys. J. C, 74, 2765

Li, W., \& Xu, L. 2014b, Eur. Phys. J. C, 74, 2870
Li, Z., Wu, P., \& Yu, H. 2009, JCAP, 09, 017

Liang, N., Xu, L., \& Zhu, Z. 2011, A\&A, 527, A11

Lu, J., Xu, L., Chang, B., Gui, Y., \& Liu, H. 2008, Phys. Lett. B, 662, 87 Luongo, O., \& Quevedo, H. 2012, Astrophys. Space Sci., 338, 345

Ma, C. P., \& Berschinger, E. 1995, ApJ, 455, 7

Moresco, M., Pozzetti, L., Cimatti, A., et al. 2016, JCAP, 05, 014 Padmanabhan, T. 2003, Phys. Rep., 380, 235

Park, C. G., Hwang, J. C., Park, J., \& Noh, H. 2010, Phys. Rev. D, 81, 063532

Peebles, P. J. E., \& Ratra, B. 2003, Rev. Mod. Phys., 75, 559

Pedram, P., \& Jalalzadeh, S. 2008, Phys. Lett. B, 659, 6

Perlmutter, S., Aldering, G., Goldhaber, G., et al. 1999, ApJ, 517, 565

Percival, W. J., Reid, B. A., Eisenstein, D. J., et al. 2010, MNRAS, 401, 2148

Piattella, O. F., Bertacca, D., Bruni, M., \& Pietrobon, D. 2010, JCAP, 01, 014

Planck Collaboration I. 2016, A\&A, 594, A1

Planck Collaboration XI. 2016, A\&A, 594, A11

Planck Collaboration XIII. 2016, A\&A, 594, A13

Riess, A. G., Filippenko, A. V., Challis, P., et al. 1998, AJ, 116, 1009

Sahni, V., \& Starobinsky, A. A. 2000, Int. J. Mod. Phys. D, 9, 373

SCP Collaboration (Amanullah, R., et al.) 2010, ApJ, 716, 712

SDSS Collaboration (Betoule, M., et al.) 2014, A\&A, 568, A22

Weinberg, S. 1989, Rev. Mod. Phys., 61, 1

WMAP Collaboration (Komatsu, E., et al.) 2011, ApJS, 192, 18

Wu, P., \& Yu, H. 2007, Phys. Lett. B, 644, 16

Xu, L. 2013a, Phys. Rev. D, 87, 043503

Xu, L. 2013b, Eur. Phys. J. C, 73, 2344

Xu, L., \& Lu, J. 2010, JCAP, 03, 025

Xu, L., Wang, Y., \& Noh, H. 2011, Eur. Phys. J. C, 72, 1931

Xu, L., Wang, Y., \& Noh, H. 2012a, Phys. Rev. D, 85, 043003

Xu, L., Lu, J., \& Wang, Y. 2012b, Eur. Phys. J. C, 72, 1883

Yang, W., \& Xu, L. 2013, Phys. Rev. D, 88, 023505 